\title{
ATLAS, and Wide-Angle Tail Galaxies in ATLAS
}

\author{
Minnie Y. Mao ${ }^{1,2,3, *}$, Rob Sharp ${ }^{2}$, D. J. Saikia, ${ }^{3,4,5}$, Ray P. Norris ${ }^{3}$, \\ Melanie Johnston-Hollitt ${ }^{6}$, Enno Middelberg ${ }^{7} \&$ Jim E. J. Lovell $^{1}$ \\ ${ }^{1}$ University of Tasmania, Hobart, 7001, Australia. \\ ${ }^{2}$ Australian Astronomical Observatory, Epping, NSW, 1710, Australia. \\ ${ }^{3}$ CSIRO Australian Telescope National Facility, Epping, NSW, 1710, Australia. \\ ${ }^{4}$ National Centre for Radio Astrophysics, TIFR, Pune 411 007, India. \\ ${ }^{5}$ International Centre for Radio Astronomy Research, University of Western Australia, \\ Crawley, WA 6009, Australia. \\ ${ }^{6}$ Victoria University of Wellington, Wellington, New Zealand. \\ ${ }^{7}$ Ruhr-Universität Bochum, 44801 Bochum, Germany. \\ *e-mail:mymao@utas.edu.au
}

\begin{abstract}
Using the Australia Telescope Compact Array (ATCA), ATLAS (Australia Telescope Large Area Survey) is imaging two fields totalling 7 square degrees down to $10 \mu \mathrm{Jy} \mathrm{beam}^{-1}$ at $1.4 \mathrm{GHz}$. We have found 6 wide-angle tail galaxies (WATs), 4 of which have sufficient data to identify associated galaxy overdensities. The largest WAT, at a red-shift of 0.22 , appears to be associated with an overdensity of galaxies that is spread over an unusually large extent of $12 \mathrm{Mpc}$, with a velocity range of $4500 \mathrm{~km} \mathrm{~s}^{-1}$. Here we present the WATs in ATLAS and discuss the implications of these observations for future large-scale radio surveys such as ASKAP-EMU.
\end{abstract}

Key words. Galaxies: clusters: general-galaxies: active-galaxies: general_radio continuum: galaxies_-galaxies: distances and red-shifts.

\section{Introduction}

The formation and evolution of galaxies over cosmic time remains one of the most perplexing issues in modern astronomy. Radio-loud galaxies can be seen to very high red-shifts, consequently deep radio surveys can be used to help understand galaxy formation and evolution. ATLAS is imaging 7 square degrees down to $\sim 10 \mu \mathrm{Jy} \mathrm{beam}^{-1}$ at $1.4 \mathrm{GHz}$ using ATCA (Data Release 1: Norris et al. 2006; Middelberg et al. 2008). To mitigate the effects of cosmic variance, ATLAS is split over two fields; Chandra Deep Field South (CDFS) and European Large Area ISO Survey - South 1 (ELAIS-S1). The ATLAS fields were chosen to coincide with the Spitzer Wide-Area InfraRed Extragalactic (SWIRE) survey program (Lonsdale et al. 2003) so that corresponding optical and infrared photometric data are available. ATLAS's first data release has an $\mathrm{rms}$ of $\sim 30 \mu \mathrm{Jy}$ beam $^{-1}$ and contains 2004 

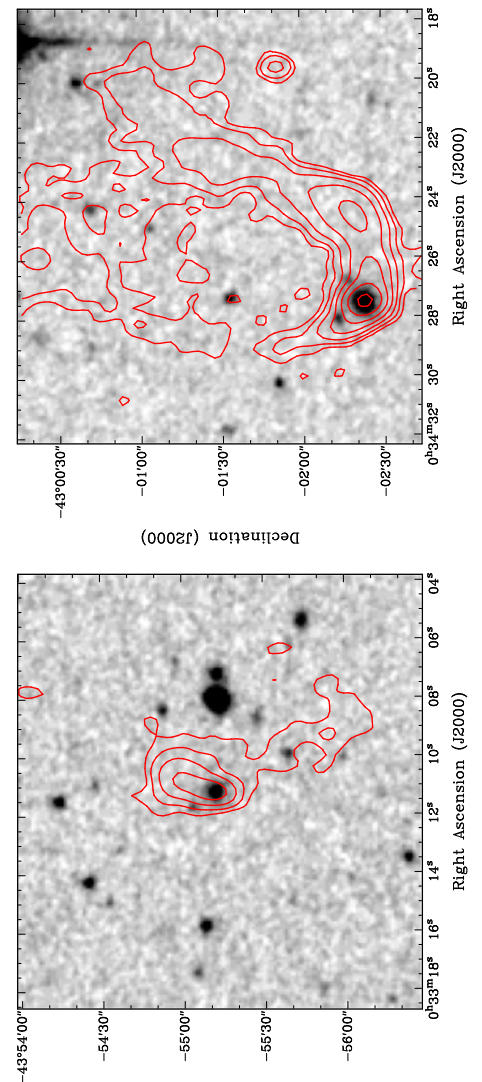

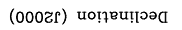

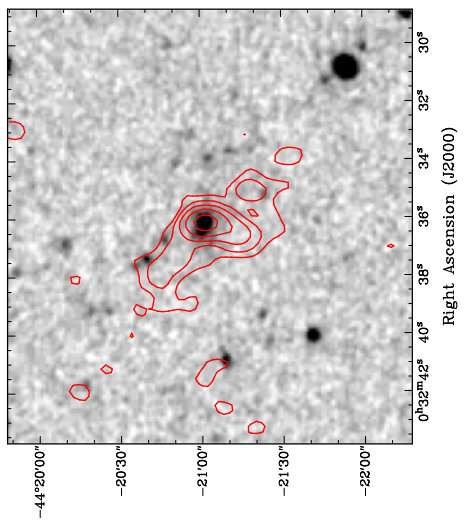

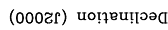
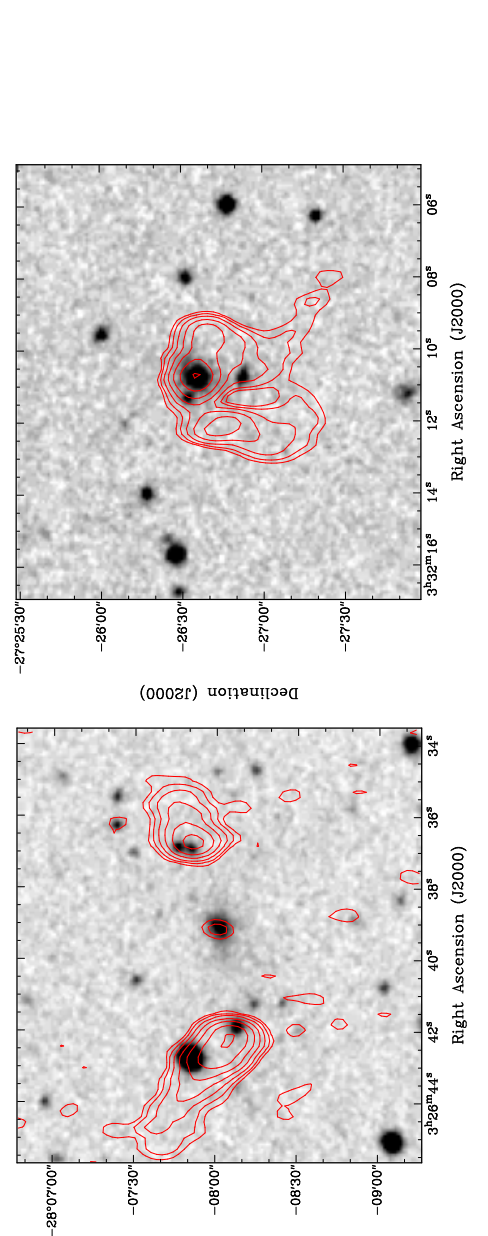

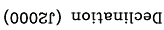

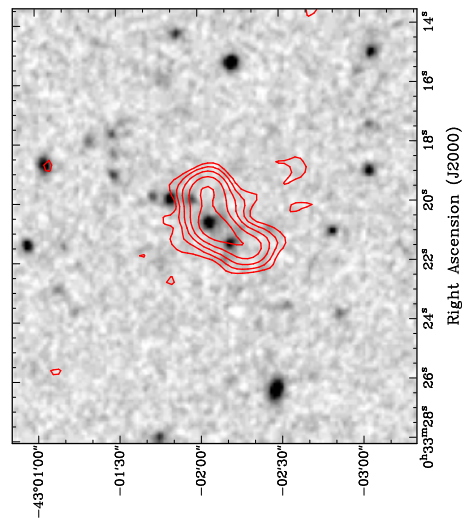

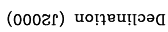


radio sources. The final data release (Banfield et al. 2011) will have an rms of $10 \mu \mathrm{Jy}$ beam $^{-1}$ and we expect the detection of 16000 radio sources. The data presented in this paper are from data release one.

The resolution of ATLAS $\left(\sim 10^{\prime \prime}\right)$ allows us to classify morphologically many of the extended radio sources including wide-angle tail galaxies (WATs) and radio relics. WATs are radio galaxies whose radio jets appear to bend in a common direction. They are usually associated with dominant cluster galaxies (Owen \& Rudnick 1976), are generally detected in unrelaxed clusters (Burns 1998) and have been used as probes for clusters (e.g. Blanton et al. 2000, 2001). In ATLAS we have found six WATs and also a putative radio relic (Middelberg et al. 2008; Mao et al. 2010).

\section{WATs in ATLAS}

We identified six WATs in ATLAS by visually examining ATLAS images. Figure 1 show the ATLAS radio contours overlaid on DSS red images. The WATs range in red-shift from 0.15 to 0.38 , and their properties are summarized in Table 1 . The WATs all have radio luminosities consistent with FRI type galaxies. The optical spectra of the host galaxies are all typical of early-type galaxies that host luminous radio sources. Four of the WATs have sufficient optical data in their immediate vicinity to allow us to probe for overdensities, and all four showed evidence for overdensities (Mao et al. 2010).

S1189, the largest WAT in our sample, appears to be associated with an overdensity that spans a linear extent of $\sim 12 \mathrm{Mpc}$. The overdensity has a velocity range of $4500 \mathrm{~km} \mathrm{~s}^{-1}$, which is similar to typical rich clusters in the local Universe undergoing mergers. There also appears to be a radio relic in the immediate vicinity ( $\sim 10$ arcmin) of S1189. In Mao et al. (2010) we discuss the possibility that the large substructure surrounding S1189 represents an unrelaxed system with different sub-structures interacting or merging with one another.

Table 1. The WATs in ATLAS. All of the $R$-band magnitudes are from SWIRE, except for S132 where we have listed the value from superCOSMOS since a value from SWIRE is not available. All the WAT red-shifts were obtained from our AAT observations with the exception of S409 whose red-shift was determined by Colless et al. (2001).

\begin{tabular}{ccccc}
\hline ATLAS & SWIRE Counterpart & $z$ & $\begin{array}{l}R_{\text {obs }} \\
(\mathrm{mag})\end{array}$ & $\begin{array}{l}\text { Power }_{1.4} \\
\left(10^{24} \mathrm{~W} / \mathrm{Hz}\right)\end{array}$ \\
\hline ELAIS & & & & \\
S132 & SWIRE4_J003236.18-442101.1 & 0.3762 & 18.1 & 2.58 \\
S483 & SWIRE4_J003311.21-435512.3 & 0.3164 & 18.28 & 2.16 \\
S1189 & SWIRE4_J003427.54-430222.5 & 0.2193 & 17.12 & 6.25 \\
S1192 & SWIRE4_J003320.68-430203.6 & 0.3690 & 18.92 & 4.82 \\
CDFS & & & & \\
S031 & SWIRE3_J032639.11-280801.5 & 0.2183 & 16.63 & 5.81 \\
S409 & SWIRE3_J033210.74-272635.5 & 0.1469 & 16.35 & 2.41 \\
\hline
\end{tabular}




\section{Implications for EMU and beyond}

We have found six WATs in ATLAS. Using deeper, higher resolution radio data, Dehghan et al. (2011) have identified 10 more WATs in CDFS. In the near future EMU, the Evolutionary Map of the Universe (Norris et al. 2011a, Norris 2011b), will image $75 \%$ of the sky down to an rms of $10 \mu \mathrm{Jy}_{\text {beam }}{ }^{-1}$ at $1.4 \mathrm{GHz}$ at a resolution similar to that of ATLAS. EMU expects to detect 70 million radio sources.

Extrapolating the six WATs out of 2004 sources in ATLAS suggests EMU might expect to detect $\sim 200000$ WATs. Alternatively, extrapolating the six WATs in $7 \mathrm{deg}^{2}$ of ATLAS suggests EMU might expect to detect $\sim 26000$ in its $\sim 30000 \mathrm{deg}^{2}$. The difference between these figures is caused by the difference in sensitivity between EMU and the ATLAS data release 1, and these two numbers therefore represent upper and lower bounds respectively. All we can say at present is that EMU will detect between 26000 and 200000 WATs. To narrow this number down we need to know the local luminosity function for WATs, the red-shift distribution of WATs, and how the luminosity function evolves with cosmic time, and our knowledge of each of these is limited by the small number of known WATs. The detection of tens of thousands of WATs in the Universe will be significant. Given that each WAT is associated with a cluster, EMU will be exceptional for detecting clusters and exploring their properties, particularly since the luminosity of the WATs makes them detectable out to high red-shifts. The detection of high red-shift clusters will contribute significantly to understanding the formation and evolution of large-scale structures and clusters.

\section{References}

Banfield J., et al. 2011, in prep.

Blanton, E. L., Gregg, M. D., Helfand, D. J., Becker, R. H., White, R. L. 2000, Astrophys. J., 531, 118.

Blanton, E. L., Gregg, M. D., Helfand, D. J., Becker, R. H., Leighly, K. M. 2001, Astron. J., 121, 2915.

Burns, J. O. 1998, Science, 280, 400.

Colless, M. et al., 2001, Mon. Not. R. Astron. Soc., 328, 1039.

Dehghan, S., Johnston-Hollitt, M., Mao, M. Y., Norris, R. P., Miller, N. A., Huynh, M. 2011, J. Astrophys. Astr., 32(4), 491.

Lonsdale, C. J. et al. 2003, Publ. Astron. Soc. Pacific, 115, 897.

Mao, M. Y., Sharp, R., Saikia, D. J., Norris, R. P., Johnston-Hollitt, M., Middelberg, E., Lovell, J. E. J. 2010, Mon. Not. R. Astron. Soc., 406, 2578.

Middelberg, E. et al. 2008, Astron. J., 135, 1276.

Norris, R. P. et al. 2006, Astron. J., 132, 2409.

Norris, R. P. et al. 2011a, Publ. Astron. Soc. Australia, 28, 215.

Norris, R. P. 2011b, J. Astrophys. Astron., 32(4), 599.

Owen F. N., Rudnick, L. 1976, Astrophys. J., 205, L1. 